\title{
Experimental investigation of the flame retardant and form-stable composite phase change materials for a power battery thermal management system
}

Jiangyun Zhang ${ }^{\mathrm{a}, \mathrm{b}}$, Xinxi $\mathrm{Li}^{\mathrm{a}, *}$, Guoqing Zhang ${ }^{\mathrm{a}}$, Hongwei $\mathrm{Wu}^{\mathrm{b}}$, Zhonghao Rao, Jianwei Guo ${ }^{\mathrm{d}}$, Dequan Zhou ${ }^{\mathrm{a}}$

${ }^{a}$ School of Materials and Energy, Guangdong University of Technology, Guangzhou, Guangdong 510006, China

${ }^{b}$ School of Engineering and Computer Science, University of Hertfordshire, Hatfield, AL10 9AB, United Kingdom

${ }^{c}$ School of Electric Power Engineering, China University of Mining and Technology, Xuzhou 221116, China

${ }^{d}$ School of Chemical Engineering and Light Industry, Guangdong University of Technology, Guangzhou, Guangdong 510006, China

\begin{abstract}
An efficient battery thermal management system (BTMS) will undoubtedly promote the performance and lifespan of battery packs. In this study, a novel flame-retarded composite PCMs composed by paraffin (PA), expanded graphite (EG),

* Corresponding author.

E-mail address: pkdlxx@gdut.edu.cn (X. Li).
\end{abstract}


ammonium polyphosphate (APP), red phosphorus (RP) and epoxy resin (ER) has been proposed for battery module. The thermophysical and flame retardant properties are investigated at both macro and micro levels. The results show that the proposed composite PCMs with an APP/RP ratio of 23/10 exhibit the optimum flame retardant performance. Besides, the APP/RP-based composite PCMs for 18650 ternary battery module has also been researched comparing with air cooled and PCM with pure PA modes. The experimental results indicated that the fire retardant PCMs shown significant cooling and temperature balancing advantages for battery module, leading to a $44.7 \%$ and $30.1 \%$ reduction rate of the peak temperature and the maintenance of the maximum temperature difference within $1.36^{\circ} \mathrm{C}$ at a $3 \mathrm{C}$ discharge rate for $25^{\circ} \mathrm{C}$. Even at $45^{\circ} \mathrm{C}$, the temperature uniformity can still be controlled within $5^{\circ} \mathrm{C}$. Thus, this research indicates the composite PCM had good flame retardant and form stable properties, it would be utilized in BTMS, energy storage and other fields.

Keywords: Composite phase change materials; Thermal properties; Flame retardant performance; Battery thermal management system; Ammonium polyphosphate and red phosphorus.

\section{Introduction}

Power batteries have been regarded as the crucial components of electric vehicles (EVs). Nevertheless, their thermal safety has been considered the most significant challenge that severely restricts the rapid development of EVs $[1,2]$. In particular, the long cycles or interrupted charge/discharge electrochemical processes 
at high discharge rates, overheating, and inhomogeneous temperature distributions power batteries suffering from inevitably lead to the severe performance degradation, decrease of the reliability, and earlier failure of power system [3,4]. Thus, developing an efficient battery thermal management system (BTMS) with a promoted heat dissipation property is very essential and urgent for power battery packs.

Currently, traditional thermal management system for battery module focusing on air cooling and liquid cooling technologies. However, the efficiency of natural convection air cooling system is low, and the forced air cooling system has large space demand and temperature distribution unevenly, especially at high charge/discharge rates, stressful and abuse conditions. Among various cooling technologies, the phase change materials (PCMs) cooling system was first proposed by Al-Hallaj, which has sparked some interest due to its distinct advantages such as working without extra energy consumption, easy installation, and convenience of maintenance [5-7]. Especially, PCMs can effectively absorb the large quantity of heat generated by power batteries, which can further reduce the temperature rise and maintain a prominent temperature consistency due to their high latent heat, so the PCMs cooling system could not only control the peak temperature within the reasonable range but also maintain the temperature distribution uniformly $[8,9]$. Paraffin (PA), as an organic PCMs, has been widely applied in the field of battery packs because of its desirable characteristics such as stable and chemically inert properties, self-nucleation, an ignorable super cooling effect, and low cost [10-12]. However, the leakage and inflammability of PA will inevitably aggravate fire safety 
risks, and this restricts the application prospects of PA in the energy storage field, especially the EVs $[13,14]$. Thus, it is necessary to provide the solutions to these restrictions.

To solve the leakage problem, the preparation of a shape-stabilized composite PCMs containing pure PA and supporting matrices has been recognized as a realistic and feasible approach [15-16]. The participation of different kinds of polymer substrates, including high density polyethylene (HDPE), low density polyethylene (LDPE), polyethylene, and epoxy resin (ER), can maintain the shape and eliminate the leakage phenomenon of form-stable composite PCMs during the phase change process [17-19]. Among these polymeric substrate materials, ER is an attainable supporting material with predominant chemical stability, strong adhesion, convenience for curing, and negligible shrinkage rate [20-23]. However, because of the particular chemical composition of PA and ER, PA/ER composites are easily flammable with a relative higher temperature condition, which severely restricts their further application. Hence, it is a meaningful assignment to improve the flame retardance performance of PA/ER.

Generally, adding flame retardant additives into shape-stabilized PCMs is the most common approach to improving their thermal stability and inhibiting their flammability $[24,25]$. Considering their life safety and environmental pollution problems, halogen-free flame retardants have been widely utilized. Additionally, red phosphorus (RP) amorphous powders, as traditional and typical representative flame retardants, exhibit some excellent performance characteristics such as 
non-volatilization, non-generation of corrosive gas, and good flame retardant effect [26-28]. Moreover, their fire-retardant mechanism can be divided into two stages. In the first stage, under a high temperature condition, the RP depolymerizes to white phosphorus and then forms phosphorus oxide oxygen acid, which can be overridden on the surface of substrate material, accelerating the dehydration and coking capacities. In the second stage, the formed liquid membrane and carbon layer can effectively achieve the separation of the external oxygen, volatile fuel, and heat with the internal polymer matrix, finally interrupting the burning process. Based on the aforementioned advantages and mechanisms, RP has been applied to improve the thermal stability and inhibit the flammability of composite PCMs. Ammonium polyphosphate (APP), as a commonly used expansion flame retardant, possesses some advantages including high content, N-P elements, excellent thermal stability, high fire-retardant fuel efficiency, and long-term flame retardant performance. For the case of thermal expansion, the non-volatile phosphorus and polyphosphoric acid cover the surface of the substrate, isolating the air to achieve flame retardation due to the nitrogen element. The APP is decomposed by heating to release nitrogen and ammonia gases, which cannot easily burn and plays a role in the dilution of oxygen in the air, thus blocking the supply of oxygen. Related studies have investigated the influence of APP/RP flame retardants on flammability. These studies indicated that the combination of APP and RP had a significant synergistic effect that would promote carbon-forming capacity and a dense carbon layer to produce sufficient insulation, further reducing the combustion rate and heat release [29-33]. Yasemin et 
al. [34] investigated the fire performance of LDPE-based composites containing APP and RP. Tang et al. [35] examined the synergistic effects of APP and RP with EG on the flammability and thermal properties of HDPE/ethyl vinyl acetate (EVA) blends. Henrik et al. [36] studied the residue stabilization in the fire retardancy of wood-plastic composites including APP, EG, and RP. Referring to the application of flame-retarded composite PCMs for cooling battery packs, Weng et al. [37] analyzed the thermal runaway propagation in the power battery modules using aerogel felt coupled with flame-retarded PCMs.

Until now, aiming to improve the thermal safety for power battery module, the flame retardant materials without PCMs (e.g. heat insulation cotton, aerogel, sealant and glass fibers) $[38,39]$ had been utilized, which could only insulate heat transmission and inhibit the spread of thermal runaway (TR). However, controlling the maximum temperature below the reasonable temperature and maintaining the temperature difference uniformly among the modules should be considered in the practical application, which play an important role in the promotion of the electrochemical performance and lifespan. Thus, it is necessary to explore a high-performance flame-retarded composite PCMs for battery modules, especially for large-scale power battery systems.

Based on the aforementioned studies, it is worthy to develop a novel composite PCMs with excellent flame retardant properties to improve the thermal safety performance and reliability of battery modules. Hence, in this research, a novel flame retardant composite PCMs based on PA/EG/APP/RP/ER was proposed. The PA 
served as the latent heat storage material, the EG served as the highly conductive additive to enhance the thermal conductivity, the ER acted as the mold component to strengthen the machinability of the composite PCMs, and the APP and RP were employed as synergistic flame retardants to improve the flame retardant performance. The thermophysical performance containing of melting point, latent heat, thermal conductivity and thermal stability, and flame retardant property of composite PCMs with different mass fractions of APP/RP flame retardants were investigated.

Furthermore, the structure and morphology of char residues were characterized with scanning electron microscopy (SEM). In addition, compared with air-cooled and pure PA modules, a PA-based flame retardant composite PCMs with the optimal proportion for a ternary battery module was assembled to carry out thermal management evaluation. According to the results, the prepared flame retardant composite PCMs had the characteristics of improved thermal stability and flame retardation, which could bring about very promising applications in thermal energy storage and thermal management.

\section{Experimental design}

\subsection{Raw Materials and preparation of the composite PCMs}

The PA was supplied by Shanghai Joule Wax. Co., Ltd. China (Purity: 99.99\%,

Melting point: $48^{\circ} \mathrm{C}$, Latent heat: $223 \mathrm{~J} / \mathrm{g}$, Thermal conductivity coefficient: 0.24 $\mathrm{W} / \mathrm{m} \cdot \mathrm{K})$. The EG could be obtained with a high temperature expansion furnace (heated at $800^{\circ} \mathrm{C}$ for $60 \mathrm{~s}$ ). Crystalline flake graphite was purchased from Qingdao 
Nansha Development Graphite Co., Ltd. China. The micro-coated RP was provided by Dongguan Zhongte Plastic Materials Co., Ltd. China. The APP was purchased from Jinan Taixing Fine Chemicals Co., Ltd. China. The ER was obtained from Changsha Baxiongdi Co., Ltd. China (with an ER-A and ER-B mass ratio of 1:1).

Traditional physical mixing and dispersing methods were employed during the preparation process of the flame retardant composite PCMs, as shown in Fig. 1. The particular preparation procedures were performed as follows: (1) The quantified amount of industrial-grade solid PA droplets were poured into a water bath kettle at the constant temperature of $70^{\circ} \mathrm{C}$ for nearly $1 \mathrm{~h}$ until completely melted. (2) The EG was added into the prepared liquid PA under mechanical agitation at $2000 \mathrm{rpm}$ for 30 min. (3) The quantified amount of ER-A was mixed with the prepared PCMs under magnetic stirring at $2500 \mathrm{rpm}$ for $10 \mathrm{~min}$. (4) The RP and APP were dispersed sequentially into the obtained liquid mixture under magnetic stirring at $2500 \mathrm{rpm}$ for 10 min. (5) The ER-B was finally poured into the mixture under magnetic stirring at $2500 \mathrm{rpm}$ for $30 \mathrm{~min}$. (6) The resulting mixture was poured into the mold with a size of $100 \times 100 \times 5 \mathrm{~mm}^{3}$ and molded in the high-low-temperature testing chamber at $80^{\circ} \mathrm{C}$ for $24 \mathrm{~h}$, which finally produced the eutectic solid flame retardant form-stable PCMs. The different components of the prepared flame-retarded composite PCMs are presented in Table $\mathrm{S} 1$. 


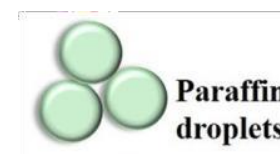

EG
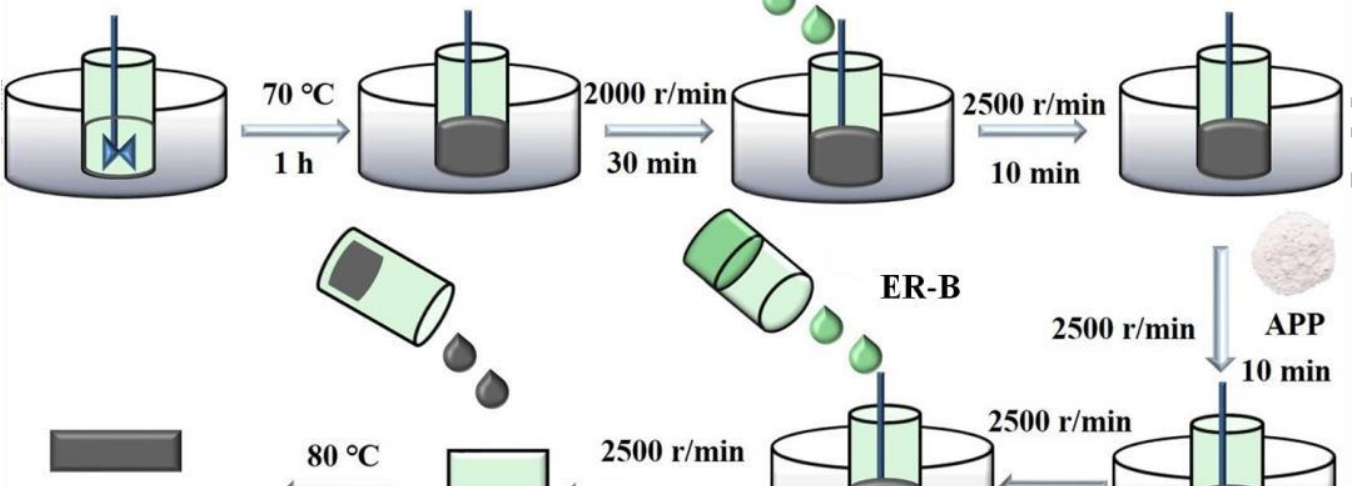

Composite PCM
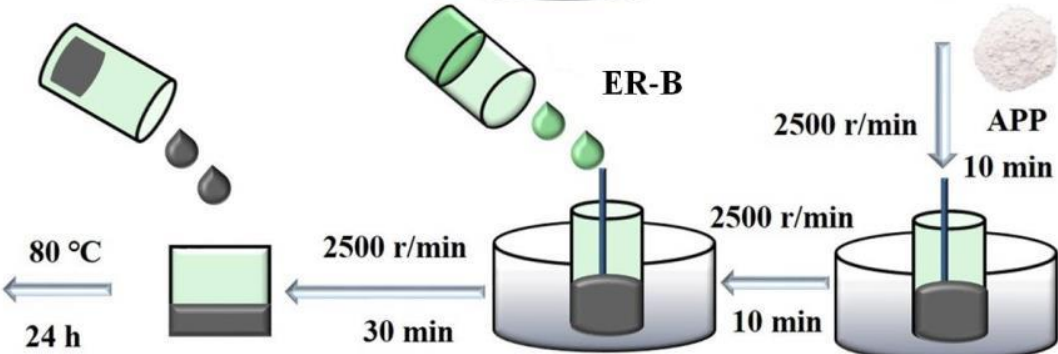

Fig. 1. Schematic diagram showing the preparation of flame-retarded composite PCMs

\subsection{Establishment of the heat dissipation model}

\subsubsection{Assembly of the battery module}

In practical, electric vehicles (EVs), the battery packs with hundreds of single batteries connecting in parallel and series should be constructed, which could satisfy the power and driving range requirement. The performance consistency among the battery module was an important factor, which would not only affect its energy density and service span but also bring about early degradation of batteries. Voltage, state of charge (SOC) and internal resistance were regarded as the most common consistency parameters, and mostly widely used in the practical battery module assembly process Thus, the electrochemical performance consistency parameters including capacity and internal resistance should be measured before the module assembling process. For the battery module with the 
aforementioned flame retardant form-stable PCMs, the detailed assembly procedures were shown in Fig. 2(a), the commercial 18650-type ternary power batteries from the same batch provided by Shenzhen Baoshengli Technology Co., Ltd. China, which were initially used for formatting and grading measurement through the battery testing system. The maximum difference of voltage and internal resistance among the batteries in the $1 \mathrm{~S} * 9 \mathrm{P}$ module should be maintained within $5 \mathrm{mV}$ and $5 \mathrm{~m} \Omega$, respectively. And the honeycomb-structure PCMs matrix with nine uniformly distributed holes (each with a diameter of $18.5 \mathrm{~mm}$ and a height of $65 \mathrm{~mm}$ ) was fabricated. In addition, the selected power batteries with arranged thermocouples were inserted into the as-prepared PCMs matrix. And then, the flexible insulation gaskets (size: $74 \times 74 \times 3 \mathrm{~mm}^{3}$ ) with nine holes with a diameter of $18.5 \mathrm{~mm}$ were affixed to the surface of the PCMs module. After that, the battery module consisting of nine 18650-type batteries was connected in 1Sx 9P configuration with nickel sheets by using a laser welding machine. Finally, the battery module was placed into the insulation shell and the resulting heat dissipation model was successfully constructed. The layout of the thermocouples (OMEGA type TT-T-30-SLE-1M, Norwalk, CT, USA; accuracy of $\pm 0.1^{\circ} \mathrm{C}$ ) is described in Fig.2(b). The $T_{5}$ located in the core of the battery modules represented the $T_{\max }$, and the $T_{9}$ located on the edge of the modules represented the $T_{\min }$. It's worth mentioning that the concrete assembly procedure and thermocouples arrangement of pure PA battery module were similar to that of aforementioned fire retardant PCMs module. The technical parameters between 
the natural convection, pure PA and the flame retarded PCMs-cooled battery modules are described in Table S2. The energy density of the three battery modules using different cooling modes can be calculated according to Formula (1). As shown in Table S2, the energy density of battery modules presented a declining trend, decreasing from $144 \mathrm{Wh} / \mathrm{kg}$ to $105 \mathrm{Wh} / \mathrm{kg}$ owing to the weight increase of battery module. Although it was an inevitable phenomenon because of the participation of composite materials, the PCMs would effectively carry out thermal management and promote the thermal safety for battery module

$$
\mathrm{W}=\frac{\mathrm{UC}}{\mathrm{m}}
$$

In Equation (1), $\mathrm{W}$ stands for the energy density, $\mathrm{Wh} / \mathrm{kg}$; $\mathrm{U}$ is the nominal voltage, $\mathrm{V} ; \mathrm{C}$ is the nominal capacity, $\mathrm{Ah} ; \mathrm{m}$ is the mass of the battery module, $\mathrm{kg}$.

(a)

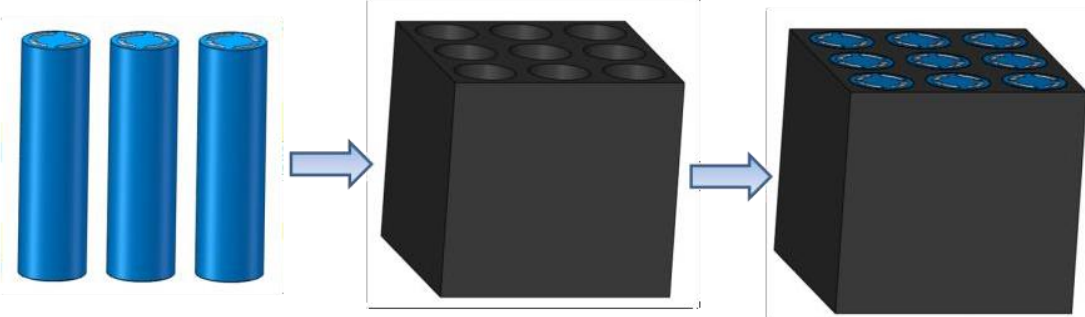

18650 cylindrical cells Composite PCM matrix Cells inserted into the specific holes

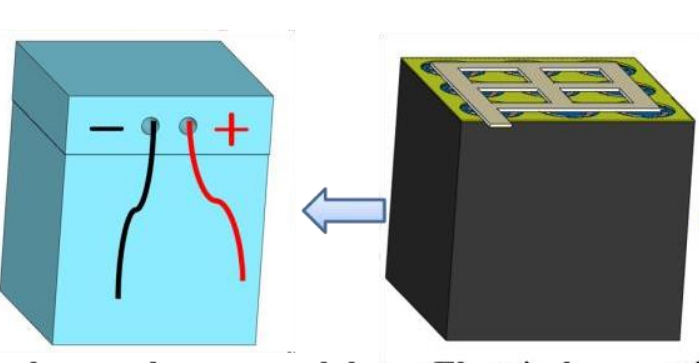

Electrical connection

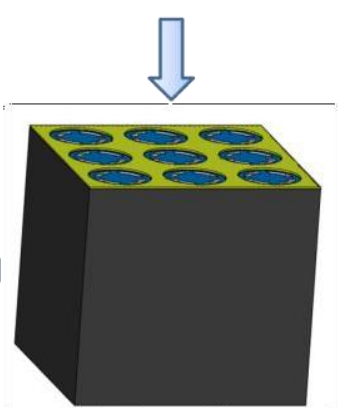

Installation of insulation gaskets 
(b)

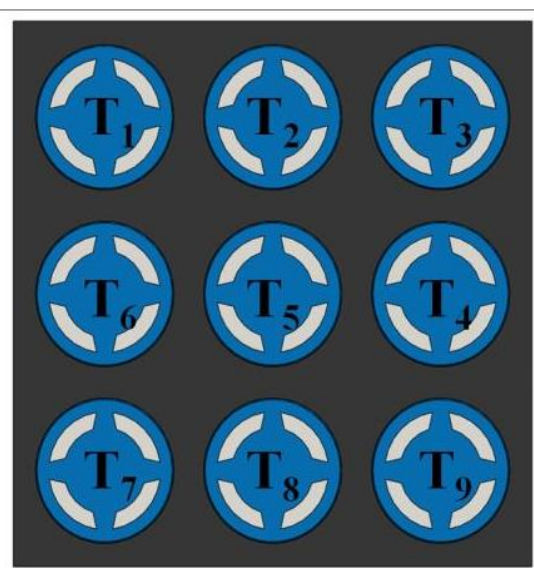

Fig. 2. Particular design scheme of the PCMs module: (a) assemble procedures and (b) layout of the thermocouples

\subsubsection{Experimental}

Particular settings of the charge-discharge experiments of battery modules are described in Fig. 3. The schematic diagrams of the experimental setup is presented in Fig. 3(a), the air-cooled and flame retardant PCMs cooling as thermal management methods for battery modules are displayed in Fig. 3(b) and Fig. 3(c), respectively. The battery modules were enclosed in a programmable thermostat $(\mathrm{BTH}-80 \mathrm{C}$, Dongguan Bell Experiment Equipment Co., Ltd. China, temperature range: $-40^{\circ} \mathrm{C}-150^{\circ} \mathrm{C}$ ) with the constant-temperature conditions of $25^{\circ} \mathrm{C}$ and $45^{\circ} \mathrm{C}$ to perform charge/discharge experiments through a battery testing system (BTS-50 V/120 A-NTF, Shenzhen Xinwei Co., Ltd. China). Additionally, nine T-type thermocouples were adhered to the surface center of each battery and connected to a temperature acquisition instrument (Agilent 34970 A, Agilent Technologies Inc. China) for testing the real-time thermal responses with the interval of $1 \mathrm{~s}$ during the entire discharge process. Then, the 
battery modules were operated according to the following charge protocol: galvanostatic mode at $1.0 \mathrm{C}$ rate with a voltage cutoff limit of $4.2 \mathrm{~V}$ and then potentiostatic mode until the current dropped to a current cutoff limit of $238 \mathrm{~mA}$. After the charging process, the modules were left to cool to ambient temperature for an adequate equilibrium time of at least $40 \mathrm{~min}$. Eventually, the battery modules were discharged at $1.0 \mathrm{C}, 2.0 \mathrm{C}$, and $3.0 \mathrm{C}$ rates in constant-current mode until the voltage declined to the cutoff limit of $2.75 \mathrm{~V}$ under the environment temperature conditions of $25^{\circ} \mathrm{C}$ and $45^{\circ} \mathrm{C}$. The particular experimental procedures of three battery modules using air, pure PA and composite flame retarded PCMs were similar.

(a)

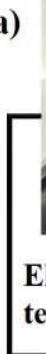

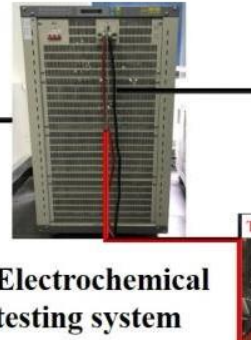

$\square$

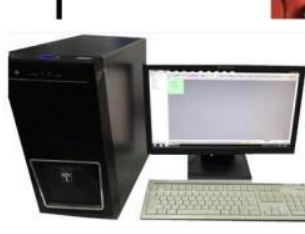

Data analysis system

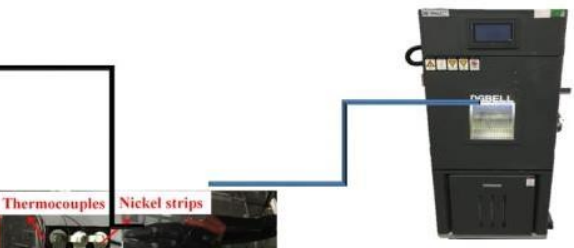

Calorstat (b)

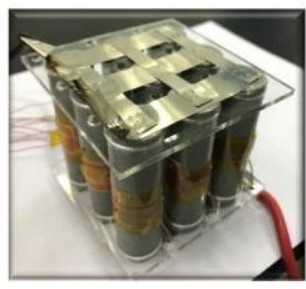

(c)

T-type thermocouples

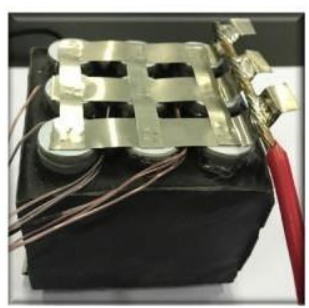

Fig. 3. Particular settings of the charge-discharge experiments: (a) schematic diagram of the battery module experimental system, (b) air-cooled battery module, and (c) battery module using the flame retarded PCMs with APP/RP enhanced

\subsection{The performances testing and characterization}

\subsubsection{Thermophysical properties analysis}


The phase change temperature, latent heat, and thermal-conducting coefficient were regarded as the most fundamental but crucial physical property parameters for the composite PCMs.

Differential scanning calorimetry (DSC, HS-DSC-101B, HESON Instrument Inc., Shanghai, China) was employed to measure the melting point and the latent heat. The experiment was carried out from $20^{\circ} \mathrm{C}$ to $70^{\circ} \mathrm{C}$ at a heating rate of $5^{\circ} \mathrm{C} / \mathrm{min}$ in the nitrogen atmosphere with a flow velocity of $20 \mathrm{~mL} / \mathrm{min}$.

The thermal diffusion coefficient was measured with an LFA447 NanoFlash ${ }^{\mathrm{TM}}$ system (range: $0.1-2000 \mathrm{~W} / \mathrm{m} \cdot \mathrm{K}$, accuracy: $\pm 5 \%$, repeatability: $\pm 3 \%$ ) with a standard rectangular sample size of $10 \times 10 \times 2 \mathrm{~mm}^{3}$. Furthermore, the thermal conductivity coefficient was calculated using Formula (2). It should be mentioned that the specimens needed to be polished to be as smooth as possible to reduce the thermal contact resistance.

$$
\lambda=\alpha \times \mathrm{C}_{\mathrm{p}} \times \rho
$$

$\lambda=$ thermal conductivity coefficient $(\mathrm{W} / \mathrm{m} \cdot \mathrm{K})$

$\alpha=$ thermal diffusion coefficient $\left(\mathrm{m}^{2} / \mathrm{s}\right)$

$\mathrm{C}_{\mathrm{p}}=$ specific heat capacity $(\mathrm{J} / \mathrm{Kg} \cdot \mathrm{K})$

$\rho=\operatorname{density}\left(\mathrm{Kg} / \mathrm{m}^{3}\right)$

\subsubsection{Thermal stability analysis}

A simultaneous DSC-thermal gravimetric analysis (TGA, SDT 2960, America TA Instrument Co., Ltd., New Castle, DE, USA) was applied for the thermal stability 
research. The samples were heated from $30^{\circ} \mathrm{C}$ to $800^{\circ} \mathrm{C}$ at a temperature rising rate of $10^{\circ} \mathrm{C} /$ min under nitrogen atmosphere protection with a gas flow velocity of 20 $\mathrm{mL} / \mathrm{min}$.

\subsubsection{Flame retardant characteristics testing}

The flame retardant properties of the composite PCMs mainly consisted of the limiting oxygen index (LOI), UL-94 rating, and cone calorimeter (CONE) measurements.

The LOI was measured by employing the oxygen index apparatus (PDF-60B, Shandong Drick Instruments Co., Ltd. China) in a mixture of oxygen and nitrogen atmosphere. The standard sizes of the samples were $120 \times 6.5 \times 3.2 \mathrm{~mm}^{3}$. It should be noted that there were at least 15 samples of each component for testing.

UL-94 experiment was conducted on a horizontal and vertical combustion tester (CZF-5, Nanjing Shineray Instruments Co., Ltd. China) under the normal air atmosphere. The standard dimensions of the specimens were $125 \times 13 \times 3.2 \mathrm{~mm}^{3}$, and at least six samples of each component were prepared. As we knew, the UL-94 burning ratings were divided into three levels containing $\mathrm{V}-0, \mathrm{~V}-1$, and $\mathrm{V}-2$, and $\mathrm{V}-0$ had the best rank.

The heat release and gas production of the composite PCMs were executed by using the CONE (FTT0007, Shenzhen Fire Testing Technology Co., Ltd. China) in the normal air atmosphere under the heat flow of $35 \mathrm{~kW} / \mathrm{m}^{2}$. Two prepared samples of each component with the approximate dimensions of $100 \times 100 \times 3 \mathrm{~mm}^{3}$ were wrapped 
in an iron container for the experiment.

\subsubsection{SEM}

The microstructure of the char residues was examined via SEM (Hitachi S-3400 $\mathrm{N}$, Tokyo, Japan) with an accelerating voltage of $20 \mathrm{kV}$. Notably, the char residues needed to be coated with gold/palladium alloy to make them ready for photography.

\section{Results and discussion}

3.1 LOI and UL-94 grading measurement

Table 1 LOI and UL-94 experiment results for the flame-retarded PCMs

\begin{tabular}{ccccccccc}
\hline Specimens & PCM 1 & PCM 2 & PCM 3 & PCM 4 & PCM 5 & PCM 6 & PCM 7 & PCM 8 \\
\hline LOI/\% & 18.4 & 22.4 & 23.8 & 24.8 & 25.4 & 27.6 & 26 & 25.6 \\
UL-94 & -- & -- & -- & -- & V-1 & V-0 & V-2 & -- \\
\hline
\end{tabular}

The results from the LOI and UL-94 rating tests of flame-retarded composite PCMs samples with varying proportions of APP/RP fire retardants are presented in Table 1. As displayed in Table 1, the LOI value of PCM 1 with virgin materials was only $18.4 \%$, and it failed to pass the UL-94 grading measurement. Nevertheless, with the increase of the APP and the reduction of the RP, the LOI of PCM 6 drastically rose to $27.6 \%$, which was the maximum value. Additionally, PCM 6 (with the APP/RP mass ratio of 23/10) passed the UL-94 V-0 rank experiment, which could be 
attributed to two major reasons: (1) There was a certain proportion of the APP that reacted with the RP to form a dense carbonization interface layer, which effectively isolated oxygen and heat from the organic materials during combustion. (2) Furtherly, the amount of APP and RP played a synergistic role in flame retardation process. At the same time, a layer of higher strength diaphragm covering the surface of the material formed during the RP burning process, which effectively prevented the fuel drop and achieved an admirable flame retardant effect. To the surprise of the authors, the LOI of PCM 7 (with the APP/RP mass ratio of 28/5) then dropped to $26 \%$.

Furthermore, the LOI of PCM 8 was reduced to $25.6 \%$ when there was only APP flame retardant. Based on the aforementioned phenomenon of LOI reduction, there was a large amount of gas production that could destroy the carbonized layer. Hence, the capabilities of separating the oxygen and heat would be greatly decreased, finally leading to the flame retardant property severely fading.

\subsection{Thermal physical properties of the composite PCMs}

(a)

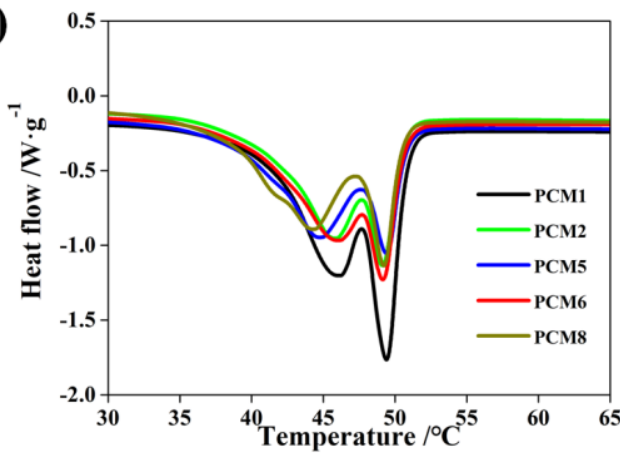

(b)

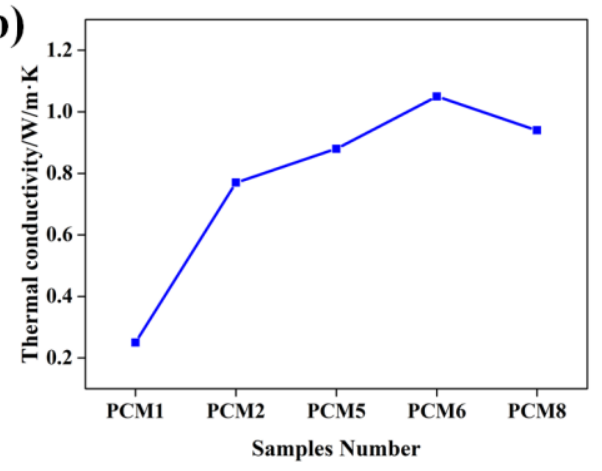

Fig. 4. Thermal characteristics of the composite PCMs: (a) DSC curves and (b) thermal 
conductivity coefficient variations.

The measurement data, as illustrated in Fig. 4(a), showed that the melting point of the flame-retarded composite PCMs was maintained in the range of $45^{\circ} \mathrm{C}-48^{\circ} \mathrm{C}$. The value was nearly close to the phase change temperature of pure $\mathrm{PA}\left(48^{\circ} \mathrm{C}\right)$, which indicated that the PA could maintain a relatively stable structure without the supererogatory chemical reactions that occurred in the preparation process of the composite PCMs. In addition, the mass fraction of the PA obviously affected the latent heat of the composite PCMs. The actual latent heat value $\left(\Delta \mathrm{H}_{\mathrm{t}}\right)$ was obtained according to Formula (3).

$$
\Delta \mathrm{H}_{\mathrm{t}}=\mathrm{f} \cdot \Delta \mathrm{H}_{\mathrm{p}}
$$

$$
\begin{aligned}
& \Delta \mathrm{H}_{\mathrm{t}}=\text { actual latent heat value }\left(\mathrm{J} \cdot \mathrm{g}^{-1}\right) \\
& \mathrm{f}=\text { mass proportion of pure PA }(\%)
\end{aligned}
$$

$$
\Delta \mathrm{H}_{\mathrm{p}}=\text { theoretical latent heat value }\left(\mathrm{J} \cdot \mathrm{g}^{-1}\right)
$$

Therefore, the latent heat of all the composite PCMs reached $89.2 \mathrm{~J} / \mathrm{g}$ because it contained a $40 \%$ proportion of PA. However, the latent heats of PCM 1, PCM 2, PCM 5, PCM 6, and PCM 8 came to $92.5 \mathrm{~J} / \mathrm{g}, 77.5 \mathrm{~J} / \mathrm{g}, 79 \mathrm{~J} / \mathrm{g}, 81.2 \mathrm{~J} / \mathrm{g}$, and $80.5 \mathrm{~J} / \mathrm{g}$, respectively, which revealed that the flame retardant PCMs showed a slightly lower value. There were three reasons providing an explanation based on the aforementioned phenomenon. Firstly, there was a relatively limited cross-linked structure of the ER to support the PA, resulting in the loss of the latent heat of the storage materials. Secondly, the thermal motions of the PA molecules during the phase 
change process were restricted by a large number of flame retardant particles. Thirdly, the thermal conductivity was obviously improved (as shown in Fig. 4(b)) owing to the participation of EG as the high thermal-conductive additives. Thus, PCM 6 exhibited the best heat transfer performance and relatively high latent heat value.

\subsection{Thermal degradation behaviors of the composite PCMs}

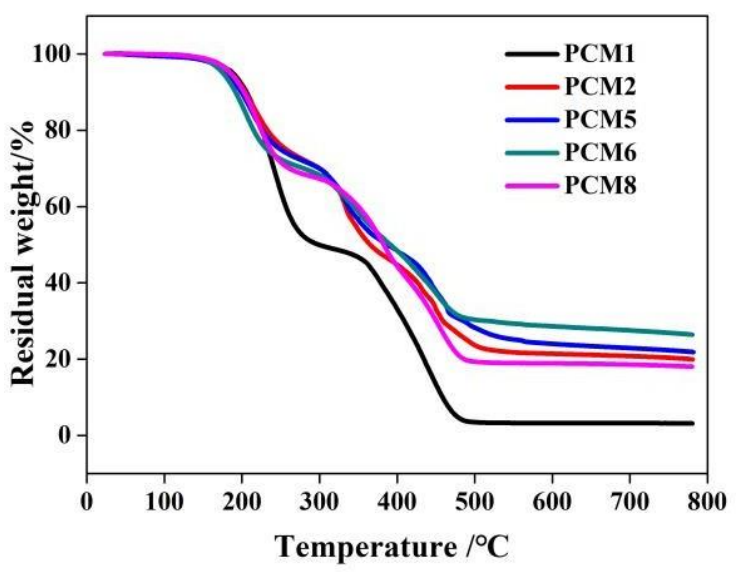

Fig. 5. TGA curves of the composite PCMs

Table 2 Core thermal stability parameters of the composite PCMs

\begin{tabular}{ccccc}
\hline Sample & $\mathrm{T}_{-5 \%}{ }^{\mathrm{a}}\left({ }^{\circ} \mathrm{C}\right)$ & $\mathrm{T}_{-25 \%}{ }^{\mathrm{b}}\left({ }^{\circ} \mathrm{C}\right)$ & $\begin{array}{c}\mathrm{T}^{\mathrm{c}}\left({ }^{\circ} \mathrm{C}\right) \\
\mathrm{p}\end{array}$ & Char residue at \\
& 188 & 233 & 489 & $600^{\circ} \mathrm{C}$ \\
\hline PCM 1 & 186 & 256 & 496 & $18.5 \%$ \\
PCM 2 & $186 \%$ & 563 & $24.8 \%$ \\
PCM 5 & 180 & 250 & 572 & $27.2 \%$ \\
PCM 6 & 179 & 270 & 514 & $21.3 \%$ \\
PCM 8 & 185 & 237 & & \\
& & 19 & &
\end{tabular}


63

64 
${ }^{a}$ indicates the temperature of the composite PCMs with a 5\% weight loss. ${ }^{\mathrm{b}}$ indicates the temperature of the composite PCMs with $25 \%$ weight loss. ${ }^{c}$ indicates the initial temperature of the final degradation platform.

The TGA curves of composite PCMs are illustrated in Fig.5, and the correlative experiment data are listed in Table 2. As described in Fig. 5, the TGA curves displayed two degradation plateaus for the composite PCMs. The first one occurred from about $150^{\circ} \mathrm{C}$ to $350^{\circ} \mathrm{C}$ due to the gasification and pyrolysis of the PA. The second stage appeared from $350^{\circ} \mathrm{C}$ to $500^{\circ} \mathrm{C}$ because of the ER degradation. Because of the involvement of APP/RP flame retardants, the thermal stability was greatly improved to a certain degree, and the PCM 6 exhibited the best value. In addition, as shown in Table 2, the $\mathrm{T}_{-5 \%}$ values of PCM 5 and PCM 6 with APP and RP flame retardants were slightly lower than those of PCM 2 and PCM 8. This might have been caused by the synergistic effect between the APP and RP accelerating the early decomposition. Furthermore, in combination with the $\mathrm{T}_{-25 \%}$ and $\mathrm{T}_{\mathrm{p}}$ PCM 2, PCM 5, and PCM 6 showed relatively higher decomposition temperatures due to the participation of the flame retardants. Additionally, the char residue of PCM 6 at $600^{\circ} \mathrm{C}$ reached the maximum value of $27.2 \%$, implying a superior charring ability and ulterior advantage. The main reason for this should be attributed to the outstanding synergistic flame retardant properties of the APP and RP. It could be inferred that the combination of the APP and RP promoted the formation of a carbon layer during the combustion process, which effectively prevented oxygen, heat, and low-molecular volatilization and restrained the combustion process. The significant synergistic effect 
of the APP and RP would be beneficial for charring, which could form a tighter carbon layer and an interfacial barrier and then decrease the combustion speed and reduce the heat release, furtherly contributing to the enhanced flame retardance of the form-stable composites PCMs.

\subsection{Cone calorimeter analysis}

(a)

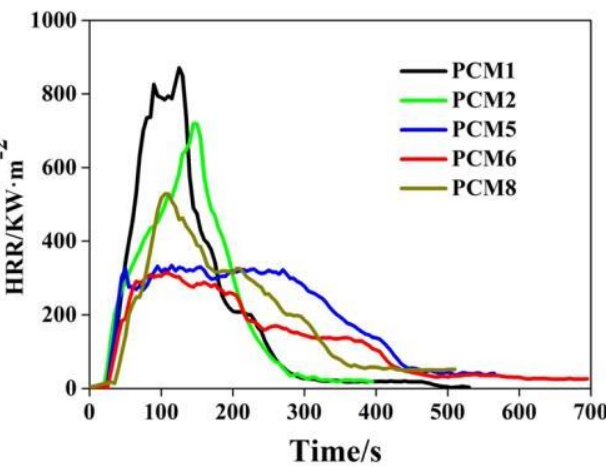

(c)

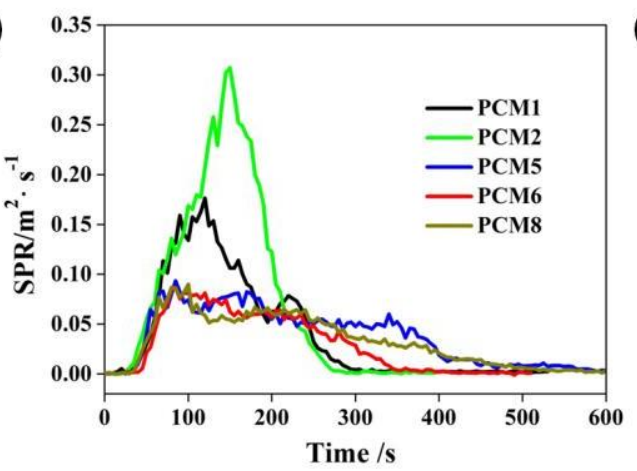

(b)

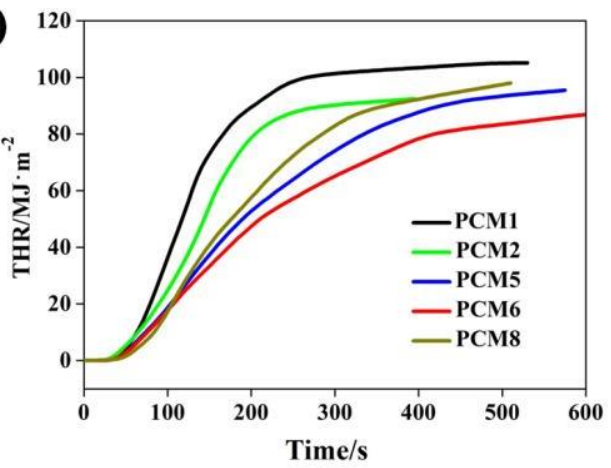

(d)

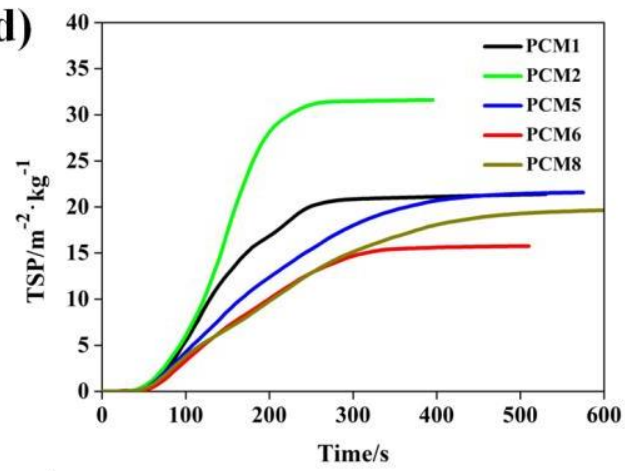

Fig. 6. CONE testing results of the composite PCMs: (a) Heat release rate (HRR), (b) Total heat release (THR), (c) Smoke production rate (SPR) and (d) Total smoke production (TSP)

To simulate the actual fire conditions, the CONE was employed to analyse the fire resistance of the five specimens through evaluating the HRR, THR, SPR, and TSP. The corresponding testing results are exhibited in Fig. 6. The HRR and the peak heat release rate (PHRR) were regarded as the important parameters closely 
reflecting the flame retardant performance.

It can be determined from Fig. 6(a) that the PHRR of PCM 1 without the involvement of flame retardant reached the maximum value of $870.9 \mathrm{~kW} / \mathrm{m}^{2}$ at nearly 125 s. However, the PHRR of composite PCMs with APP/RP synergistic flame retardant displayed a declining trend, which came to $719.6 \mathrm{~kW} / \mathrm{m}^{2}, 334.2 \mathrm{~kW} / \mathrm{m}^{2}$, $313.1 \mathrm{~kW} / \mathrm{m}^{2}$, and $528.6 \mathrm{~kW} / \mathrm{m}^{2}$ for PCM 2, PCM 5, PCM 6, and PCM 8 , respectively. It should be noted that the PHRR of PCM 6 was the lowest, which was attributed to the synergistic effect between the APP and RP, which in turn resulted in the formation of the high-quality carbon layer to isolate oxygen and heat, thus preventing the PCMs from continuing burning. Additionally, Fig. 6(b) implies that the THR of PCM 6 reached the minimum value of $89.3 \mathrm{MJ} / \mathrm{m}^{2}$, which was decreased by $17.8 \%, 3.47 \%, 6.94 \%$, and $9.74 \%$ in comparison with the values of PCM 1, PCM 2, PCM 5, and PCM 8, respectively. It could be concluded that the heat releasing curves of PCM 6 exhibited a smoother and gentler tendency compared to the curves of the other samples, which effectively demonstrated that the flame retardant performance was greatly improved.

To the best knowledge of the authors, the large quantities of smoke and toxic gas produced in the combustion process are considered as the most severe potential threat to the human body $[40,41]$. Hence, the evaluation of the SPR and TSP were of great significance. From Fig. 6(c) and Fig. 6(d), the SPR and TSP of PCM 2 reached the maximizing, which were caused by the combustion of RP. However, the SPR of PCM 6 came to the lowest point, accompanied by the obviously gentle trend of the TSP 
curves. The reasons leading to the above phenomenon could be ascribed to two aspects. On the one hand, accompanied by the increase of the APP and the decrease of the RP, the SPR and TSP between PCM 5 and PCM 6 showed a declining trend because of the expanded carbon layer structure formed by the thermal decomposition and dehydration of the expanded flame retardant APP, which absorbed a small amount of flue gas released from the composite PCMs. This resulted in a smoke suppression effect. On the other hand, the excessive APP resulted in the formation of a loosened carbon structure, arising from the shortage of carbon sources in the degradation process of the APP. Thus, it could be concluded that the amount of APP and RP with an appropriate ration for PCM 6 produced a dense carbon layer, effectively separating from the flue gas due to the synergistic reaction mechanism and decreasing the potential hazard.

\subsection{Char residues analysis}
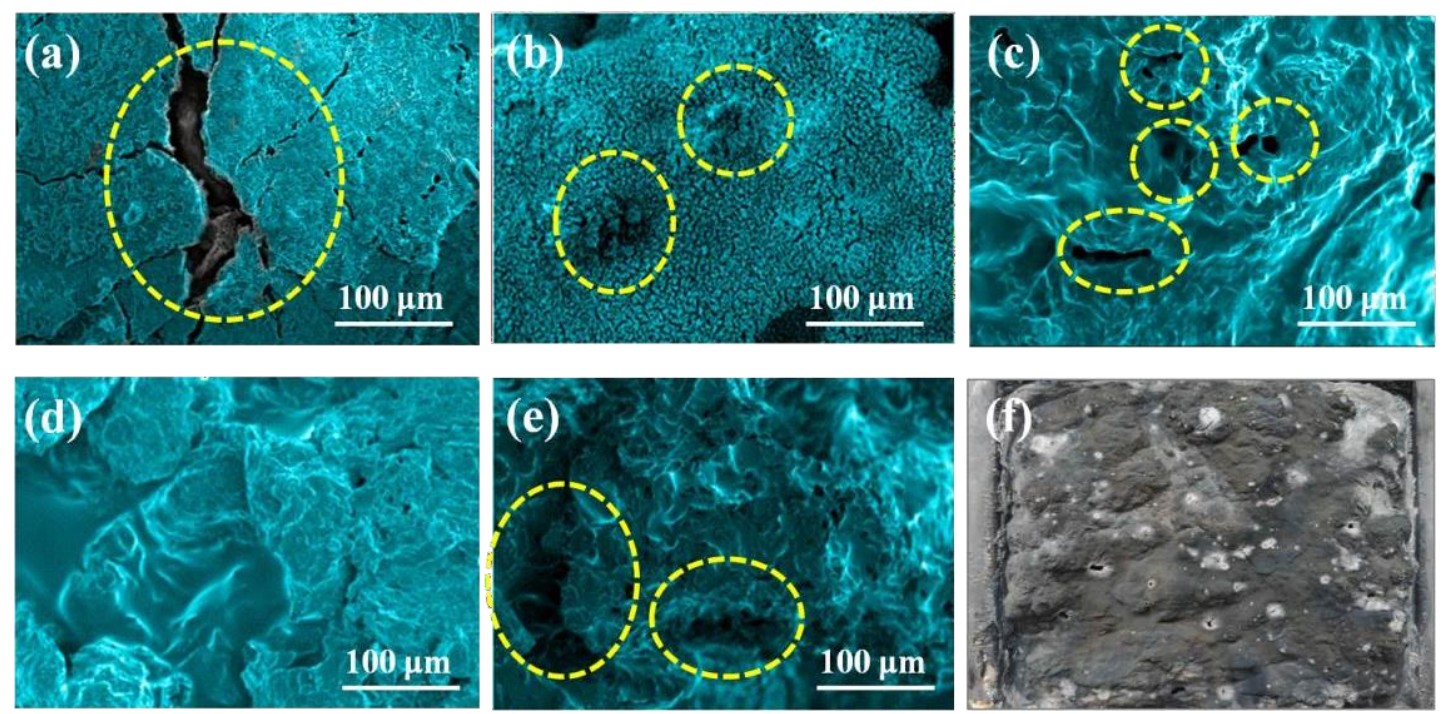

Fig. 7. The SEM images and photos of the char residues after combustion: (a) PCM 1, (b) PCM 2, 
(c) PCM 5, (d) PCM 6, (e) PCM 8 and (f) the digital photographs of PCM 6

In order to clearly reveal the flame retardant mechanism from the microscopic perspective, the digital photographs and SEM images of the char residues after the combustion were analyzed. From Fig. 7, the morphology of PCM 1 (Fig. 7(a)) was loose with large cracks due to insufficient carbon formation or less coke condensation during combustion. As shown in Fig. 7(b), the amount of carbon residue of PCM 2 without obvious cracks was relatively large, presenting a uniform porous structure. This phenomenon might be due to the flammability of the RP, which might have further damaged the carbon structure with excessive RP during the combustion stage. For the composite PCM 8 (Fig. 7(e)) with single APP adopted as the flame retardant, the surface still had a few interspaces due to the simplex carbon layer construction that resulted from the insufficient acid and gas source, which could not effectively protect the substrate and isolate the air and heat. For PCM 5, as shown in Fig. 7(c), due to the relatively small quantity of APP and the lack of the carbon source involved in carbon formation, the carbon layer that formed was further damaged due to the excessive RP in the combustion process. The digital photographs of PCM 1, PCM 2, PCM 5 and PCM 8 samples are exhibited in Fig. S2. It could be clearly seen that the carbon layer of PCM 6 (Fig. 7(f)) with a murky grey surface and low porosity was much more continuous and dense, resulting in isolation from the air and heat generated by the thermal source, which protected the matrix material and improved the flame retardant performance. Additionally, the heat generation and the char residues experienced decomposition during the combustion process, which brought 
about the carbonization and carbonization-promotion of the APP and RP and led to the improvement of the flame retardant property, which was confirmed by the continuous and compact carbon layer structure of PCM 6, as shown in Fig. 7(d).

Therefore, it could be concluded that the optimum mass proportion of the APP/RP was 23/10 in PCM 6, which exhibited a more continuous and compact carbon layer structure for effectively promoting the flame retardant property.

\subsection{Heat dissipation performance evaluation}

(a) Air cooled module Electrical insulating board

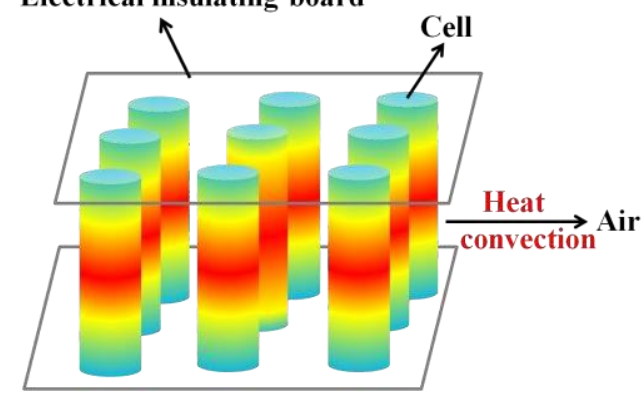

(b) PCMs cooling module

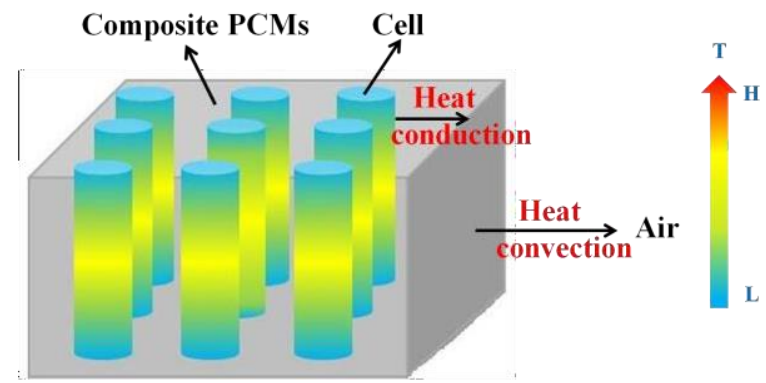


(c)

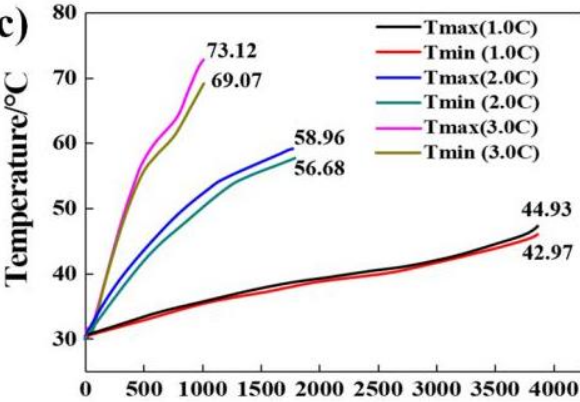
Discharge time/s

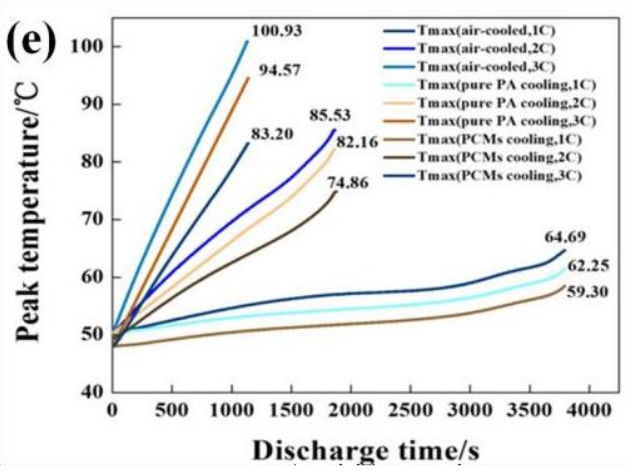

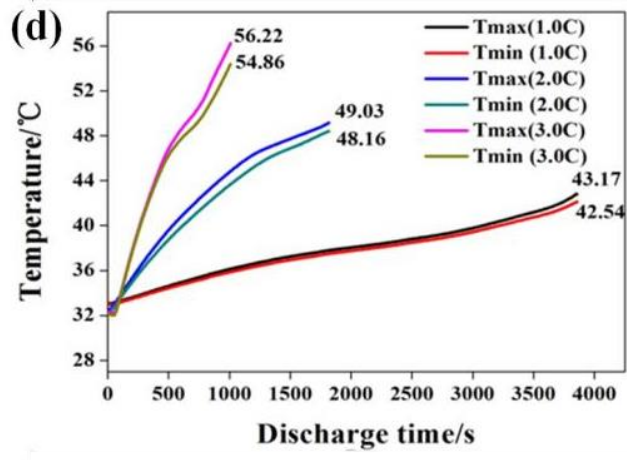

(f)

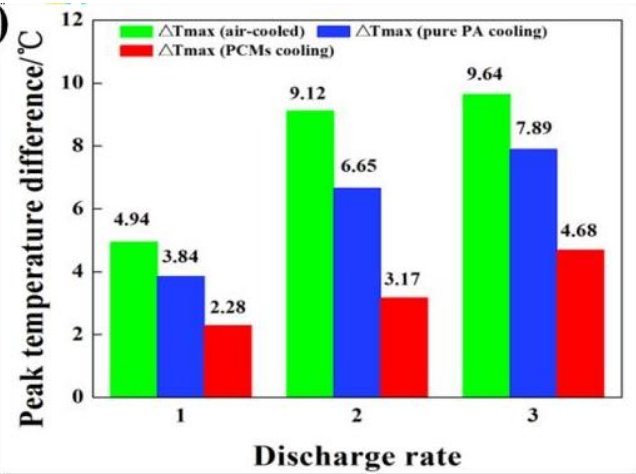

Fig. 8. Comparison of cooling effect of different BTMS: (a) heat dissipation principle of air cooling mode, (b) heat dissipation mechanism of PCMs mode, (c) temperature variations of the battery module based on pure PA cooling $\left(25^{\circ} \mathrm{C}\right)$ at different discharge rates, (d) temperature variations of the battery module employing the flame retarded PCMs $\left(25^{\circ} \mathrm{C}\right)$ at different discharge rates, (e) peak temperature $\left(45^{\circ} \mathrm{C}\right)$ and (f) the maximum temperature difference $\left(45^{\circ} \mathrm{C}\right)$

From Fig. 8(a) and Fig.8 (b), the distinctions of different BTMS using air, pure PA and the flame-retarded composite PCMs as the heat dissipation media are illustrated. As can be seen, for the BTMS using air as the heat dissipation medium, the single mode of heat dissipation was by natural air convection through the spaces between the batteries and flowing air. In contrast, for the cooling modes employing pure PA and flame-retarded PCMs-based composites (Fig. 8(b)), the heat generation that was generated by the batteries was absorbed by the PCMs in time through the 
heat conduction mode. Then, the heat in the composite PCMs was diffused by heat convection with the air, which led to the rapid decline of the peak temperature and shortened the batteries' exposure time to the high temperature ambient. In addition, the temperature distribution uniformity was furtherly improved via the direct contact between the batteries and the composite PCMs. Consequently, the integrated performance, service life and reliability of the power system were enhanced, respectively.

Moreover, PCM 6 with the optimum flame retardant property was applied in the lithium ion power battery module $(3.6 \mathrm{~V} / 19.8 \mathrm{Ah})$ to investigate the cooling effect. Testing results of air-cooled battery module were described in Fig. S3(a). As illustrated in Fig. S3(a), at 1.0 C, 2.0 C, and 3.0 C rates during discharge, the peak temperatures of the power module reached $45.82^{\circ} \mathrm{C}, 67.33^{\circ} \mathrm{C}$, and $81.35^{\circ} \mathrm{C}$, respectively in the absence of PCMs. Worse still, severe temperature heterogeneity occurred, resulting in the maximum temperature difference increasing from $4.34^{\circ} \mathrm{C}$ to $9.73^{\circ} \mathrm{C}$, which brought about the serious degradation of the electrochemical performance of the battery module and easily triggered thermal runaway. Fig. 8(c) and Fig. 8(d) described the temperature variations and temperature coherence of pure PA-based and constructed PCMs-based battery modules at different discharge rates for room temperature condition of $25^{\circ} \mathrm{C}$. Testing results of pure PA battery module (Fig. $8(\mathrm{c})$ ) indicated that the peak temperature decreased to $44.93^{\circ} \mathrm{C}, 58.96^{\circ} \mathrm{C}$, and $73.12^{\circ} \mathrm{C}$, accompanying with the maximum temperature difference of $1.96^{\circ} \mathrm{C}, 2.28^{\circ} \mathrm{C}$, and $4.05^{\circ} \mathrm{C}$ when the discharge rates were $1.0 \mathrm{C}, 2.0 \mathrm{C}$ and $3.0 \mathrm{C}$, respectively. It was 
noting that the cooling capacity was promoted to some extent owing to the participation of pure PA acting as cooling medium. In contrast, as presented in Fig. 8(d), the peak temperature of the battery module using fire retardant PCMs as heat transfer working medium dropped to $43.17^{\circ} \mathrm{C}, 49.03^{\circ} \mathrm{C}$, and $56.22^{\circ} \mathrm{C}$ with $1.0 \mathrm{C}, 2.0$ $\mathrm{C}$ and 3.0 $\mathrm{C}$ discharging rates, respectively, exhibiting the obvious declining trend compared to that of the other aforementioned two cooling modes. Furthermore, maintaining a relatively low temperature difference of all the batteries in the battery module was beneficial for stabilizing the electrochemical characteristics and prolonging the lifespan. It should be noted that participation of PCM 6 for the battery module greatly promoted the temperature consistency, and the maximum temperature difference among the batteries significantly decreased at various discharge rates. For the $3 \mathrm{C}$ discharge rate in particular, the maximum temperature difference was only $1.36^{\circ} \mathrm{C}$, which was $8.37^{\circ} \mathrm{C}$ and $2.69^{\circ} \mathrm{C}$ lower than that of the battery modules employing air-cooled and pure PA cooling modes.

In addition, in order to evaluate the heat dissipation effect of the aforementioned PCMs cooling module under harsh working conditions, such as a relative high environment temperature, further analysis was performed. Thus, the heat behaviors between the air-cooled, pure PA cooling and flame-retardant PCM 6 composites with APP/RP enhanced modules at various discharge rates were analyzed. The values of the peak temperature and maximum temperature difference are presented in Fig. 8(e) and Fig. 8(f). Even at a $3 \mathrm{C}$ discharge rate at $45^{\circ} \mathrm{C}$, the peak temperature of battery module with PCM 6 composites decreased by $21.3 \%$ and $13.7 \%$, respectively, 
compared with the values of the air-cooled and pure PA modules. What's better, the peak temperature difference came to $4.68^{\circ} \mathrm{C}$, which was $4.96^{\circ} \mathrm{C}$ and $3.21^{\circ} \mathrm{C}$ lower than that of the battery modules using air and PA as cooling mediums especially at 3.0 $\mathrm{C}$ high discharge rate, implying the greatly promoted temperature coherence. The aforementioned testing results indicated that the cooling system utilizing the flame

retarded PCMs exhibited remarkable temperature-controlling and temperature-equalizing advantages for a battery module even in abominable work conditions. Thus, it can be deduced that the application of an enhanced flame retarded PCMs cooling system for a battery module can not only control the peak temperature but also balance the temperature uniformity, revealing the outstanding heat transfer performance, especially at a high discharge rate and high temperature condition.

\section{Conclusions}

In this study, for improving the safety performance of composite PCMs for a power battery system, a novel PA-based composite flame retardants form-stable PCMs with APP/RP were proposed and applied in 18650 ternary battery module. The influence of various fractions of the APP/RP flame retardants on the thermal physical properties, thermal stability, and fire-retardant peculiarity were experimentally investigated in detail, and the morphologies and structures of the char residues after combustion were analyzed. And the optimum APP/RP proportion of the composite PCMs was selected and utilized in the battery module to improve the thermal management effectiveness. The results revealed that composite PCMs containing the 
mass ratio of 23/10 of APP/RP could exhibit a better high-quality carbon layer with a compact and homogeneous structure and obtain excellent flame retardant performance, it could achieve the highest LOI value of $27.6 \%$ and the char residue peak value of $27.2 \%$.

Comparing with the air-cooled and pure PA cooling systems, the composite flame retardants form-stable PCMs displayed better thermal management effectiveness. At a $3 \mathrm{C}$ discharge rate for a room condition of $25^{\circ} \mathrm{C}$, the peak temperature was decreased by $44.7 \%$ and $30.1 \%$, respectively. And the peak temperature difference was kept within $2^{\circ} \mathrm{C}$. Even at 3.0 C high discharge current under $45^{\circ} \mathrm{C}$ condition, the maximum temperature was reduced by $21.3 \%$ and $13.7 \%$, respectively, the temperature differences could be maintained within $5^{\circ} \mathrm{C}$. The results revealed that the PCMs cooling system had a more important role in slowing down the increase of temperature and balancing the temperature performance, especially at a high discharge rate.

Considering the above analysis, it should be concluded that the PA-based composite flame-retarded PCMs with APP and RP exhibited outstanding thermal properties, and they could not only improve the thermal management effectiveness for the ternary power battery module but also be utilized in other energy fields as potential thermal energy storage materials. Nevertheless, considering different BTMS including air cooling, liquid cooling and PCM cooling approaches had their own special characteristics, for example, the space available, cost, weight, integration degree and service-life, so it was very necessary to rationally design and install 
appropriate thermal management system according to the demand of heat dissipation in the practical loading condition.

\section{Acknowledgements}

This research was financially supported by the National Natural Science Foundation of China (Grant no. 51906047), the National Natural Science Foundation of China (Grant no. 21875046), the National Natural Science Foundation of China (Grant no.51803036) and Foshan city science and technology innovation project (2017IT100143).

\section{References}

[1] Weng JW, Ouyang DX, Yang XQ, et al. Optimization of the internal fin in a phase-change-material module for battery thermal management. Appl Therm Eng 2020;167:114698.

[2] Chen MY, Ouyang DX, Weng JW, et al. Environmental pressure effects on thermal runaway and fire behaviors of lithium-ion battery with different cathodes and state of charge. Process Saf Environ 2019;130:250-6.

[3] Weng JW, Yang XQ, Ouyang DX, et al. Comparative study on the transversal/lengthwise thermal failure propagation and heating position effect of lithium-ion batteries. Appl Energy 2019;255:113761.

[4] Gao S, Feng XN, Lu LG, et al. An experimental and analytical study of thermal runaway propagation in a large format lithium ion battery module with NCM pouch-cells in parallel. Int J Heat Mass Tran 2019;135:93-103. 
[5] Weng JW, He YP, Ouyang DX, et al. Thermal performance of PCM and branch-structured fins for cylindrical power battery in a high-temperature environment. Energy Convers Manage 2019;200:112106

[6] Ryo K, Yuta A, Yuji Y, et.al. Thermophysical properties of trimethylolethane (TME) hydrate as phase change material for cooling lithium-ion battery in electric vehicle. J Power Sources 2019;427: 70-6.

[7] Lv YF, Liu GJ, Zhang GQ, et.al. A novel thermal management structure using serpentine phase change material coupled with forced air convection for cylindrical battery modules. J Power Sources 2020;468: 228398.

[8] Weng JW, Yang XQ, Zhang GQ, et.al. Optimization of the detailed factors in a phase-change-material module for battery thermal management. Int J Heat Mass Tran 2019;138:126-34.

[9] Joshy N, Hajiyan M. Siddique ARM, et.al. Experimental investigation of the effect of vibration on phase change material (PCM) based battery thermal management system. J Power Sources 2020;450: 227717.

[10] Bamdezh MA, Molaeimanesh GR. Impact of system structure on the performance of a hybrid thermal management system for a Li-ion battery module. $\mathbf{J}$ Power Sources 2020;457: 227993.

[11] Xu T, Li Y, Chen J, et al. Improving thermal management of electronic apparatus with paraffin/expanded graphite/graphene composite material. Appl Therm Eng 2018;140:13-22.

[12] Mehdi MK, Ehsan H, Mehdi M. A novel hybrid thermal management for Li-ion 
batteries using phase change materials embedded in copper foams combined with forced-air convection. Int J Therm Sci 2019;141:47-61.

[13] Wu HY, Li ST, Shao YW, et al. Melamine foam/reduced graphene oxide supported form-stable phase change materials with simultaneous shape memory property and light-to-thermal energy storage capability. Chem Eng J 2020;379:122373.

[14] Xu L, Liu X, Yang R. Flame retardant paraffin-based shape-stabilized phase change material via expandable graphite-based flame-retardant coating. Molecules 2020;25:2408.

[15] Huang YH, Cheng YX, Zhao R, et al. A high heat storage capacity form-stable composite phase change material with enhanced flame retardancy. Appl Energy 2020;262:114536.

[16] Kazanci B, Cellat K, Paksoy H. Preparation, characterization, and thermal properties of novel fire-resistant microencapsulated phase change materials based on paraffin and a polystyrene shell. RSC advances 2020;10:24134-44.

[17] Huang YH, Cheng WL, Zhao R. Thermal management of Li-ion battery pack with the application of flexible form-stable composite phase change materials. Energy Convers Manage 2019;182:9-20.

[18] Wu WX, Wu Wei, Wang SF. Form-stable and thermally induced flexible composite phase change material for thermal energy storage and thermal management applications. Appl Energy 2019;236:10-21.

[19] Lv YF, Situ WF, Yang XQ, et.al. A novel nanosilica-enhanced phase change 
material with anti-leakage and anti-volume-changes properties for battery thermal management. Energy Convers Manage 2018;163:250-9.

[20] Naria EA, Miguel AAF, Andres SG, et al. Novel formulations of phase change materials epoxy composites for thermal energy storage. Materials 2018;11:1-18 [21] Su JF, Zhao YH, Wang XY, et al. Effect of interface debonding on the thermal conductivity of microencapsulated-paraffin filled epoxy matrix composites. Compos Part A-Appl S 2012;43:325-32

[22] Ma TT, Li LP, Wang QW, et al. High-performance flame retarded paraffin/epoxy resin form-stable phase change material. J Mater Sci 2019;54:875-85

[23] Lian QS, Li Y, Sayyed AAS, et al. Facile strategy in designing epoxy/paraffin multiple phase change materials for thermal energy storage applications. Acs Sustain Chem Eng 2018;6:3375-84.

[24] Xu L, Liu X, An ZH, et al. EG-based coatings for flame retardance of shape stabilized phase change materials. Polym Degrad Stabil 2019;161:114-20.

[25] Sebastian R, Yuttapong C, Bernhard S. Exploring the modes of action of phosphorus-based flame retardants in polymeric systems. Materials 2017;10:1-23.

[26] Ding Y, Swann JD, Sun Q, et al. Development of a pyrolysis model for glass fiber reinforced polyamide 66 blended with red phosphorus: Relationship between flammability behavior and material composition. Compos Part B-Eng 2019;176:107263.

[27] Liang JZ, Zhu B, Pan MS, et al. Melt shear flow behavior of flame-retardant polypropylene composites filled with microencapsulated red phosphorus. J 
Thermoplast Compos 2019;32:1361-77.

[28] Ji WF, Yao Y, Guo J, et al. Toward an understanding of how red phosphorus and expandable graphite enhance the fire resistance of expandable polystyrene foams. J Appl Polym Sci 2020;137:49045.

[29] Chen RJ, Huang XY, Zheng RZ, et al. Flame-retardancy and thermal properties of a novel phosphorus-modified PCM for thermal energy storage. Chem Eng J 2020;380:122500.

[30] Dong X, Yang JN, Hua XZ, et al. Synthesis of a novel char-forming agent (PEIC): Improvement in flame retardancy, thermal stability, and smoke suppression for intumescent flame-retardant polypropylene composites. J Appl Polym Sci 2020;137:1-11.

[31] Ma D, Li J. Synthesis of a bio-based triazine derivative and its effects on flame retardancy of polypropylene composites. J Appl Polym Sci 2020;137:245-53.

[32] Ribeiro SPS, Martins RC, Estevao LRM, et al. Microscopy as a tool to investigate the influence of ammonium polyphosphate particle size on the flame retardant properties of polymer composites. Microsc Res Techniq 2019; DOI: $10.1002 /$ jemt.23411

[33] Liu D, Hu AJ. The influence of environmentally friendly flame retardants on the thermal stability of phase change polyurethane foams. Materials 2020; 13:520

[34] Yasemin A, Mehmet D, Erdal B. The effect of red phosphorus on the fire properties of intumescent pine wood flour-LDPE composites. Fire Mater 2016;40:697-703. 
[35] Tang MQ, Qi F, Chen M, et al. Synergistic effects of ammonium polyphosphate and red phosphorus with expandable graphite on flammability and thermal properties of HDPE/EVA blends. Polym Advan Technol 2016;27:52-60.

[36]Henrik S, Ulrike B, Manfred HW. Residue stabilization in the fire retardancy of wood-plastic composites: combination of ammonium polyphosphate, expandable graphite, and red phosphorus. Macromol Chem Phys 2012;213:2370-77.

[37]Weng JW, Ouyang DX, Yang XQ, et al. Alleviation of thermal runaway propagation in thermal management modules using aerogel felt coupled with flame-retarded phase change material. Energy Convers Manage 2019;200:112071. [38]Huang YH, Cheng YX, Zhao R, et al. A high heat storage capacity form-stable composite phase change material with enhanced flame retardancy. Appl Energy 2020;262:114536.

[39]Li XX, Huang QQ, Deng J, et al. Evaluation of lithium battery thermal management using sealant made of boron nitride and silicone. J Power Sources 2020;451:227820.

[40]Xu L, Wang JP, Yang R. A new flame retardance strategy for shape stabilized phase change materials by surface coating. Sol Energ Mat Sol C 2017;170:87-94.

[41]Xu ZS, Yan L, Chen L. Synergistic flame retardant effects between aluminum hydroxide and halogen-free flame retardants in high density polyethylene composites. Procedia Engineering 2016;135:631-6. 